\title{
Ik-Kyung Jang (Ed): Cardiovascular OCT Imaging
}

\author{
Springer International Publishing, Switzerland, 2015, ISBN: 978-3-319-10800-1
}

\author{
Valeria Gaudieri $^{1} \cdot$ Luigi Mansi $^{1}$
}

Published online: 12 May 2016

(C) Springer-Verlag Berlin Heidelberg 2016

This text is a guide to cardiovascular use of optical coherence tomography (OCT).

The editor is Ik-Kyung Jang, Professor of Medicine, Harvard Medical School. He has pioneered the application of OCT in cardiology, establishing the OCT criteria for plaque characterization and comparing OCT and intravascular ultrasonography in living patients.

The book contains 15 chapters with a useful index, occupying 222 pages and including numerous representative illustrations.

The first two chapters focus on the development of OCT with emphasis on fundamental notions, the process of technology translation and clinical applications, the first of which was in ophthalmology, where it has become a standard of care, and then more recently in cardiology, both as a diagnostic modality and research tool.

The following chapters are devoted to OCT validation, comparing OCT images and histological findings of atherosclerotic plaque, calcification, fibroatheroma, thrombus and neointima formation, image interpretation and possible pitfalls and artifacts. However, the most interesting part centres on the clinical presentations of cardiovascular diseases, covering acute coronary syndrome and spontaneous coronary artery dissection, and on evaluation of early and late stents with regard to possible mechanisms of failure, such as malapposition, stent thrombosis and neoatherosclerosis.

In chapter XIV, the authors review the conclusion of consensus documents released and highlight the importance of reaching agreement on the best OCT definitions to describe plaque anatomy and stented segments because researchers still use different criteria and descriptions.

The last chapter focuses on future developments. Multimodal imaging is certainly a promising research field: the combinations of different modalities can provide unique information, overcoming the weaknesses of each technique alone.

Although this volume is mainly addressed to interventional cardiologists, providing background and useful hints, we recommend this fascinating book to all clinicians interested in vascular biology to improve their understanding of the role and evolution of high-risk plaque in the pathogenesis of acute coronary syndrome.
Luigi Mansi

luigi.mansi@unina2.it

1 Second University of Naples, Naples, Italy 